DOI: $10.17516 / 1997-1370-0865$

УДК 332.142.4:005.342

\title{
Empirical Assessment of the Efficiency of Sustainable Innovative Development of the Resource-based Regions
}

\author{
Irina R. Ruiga* \\ Siberian Federal University \\ Krasnoyarsk, Russian Federation
}

Received 27.10.2021, received in revised form 05.11.2021, accepted 10.11.2021

\begin{abstract}
The article is devoted to the formation of assessment tools for monitoring threats to the innovative development of the constituent entities of the Russian Federation from the perspective of criteria for efficiency and sustainability of performance. Taking into consideration investigated approaches and advantages and disadvantages identified in the course of the research, the author developed an assessment approach of the efficiency of the regional sustainable innovative development from the perspective of sustainability. The assessment mechanism is based on the calculation of the integral index for two projections (innovative potential and innovative development efficiency). Testing of the current approach is implemented on the example of the resource-type regions of the Russian Federation. The research results can be used by regional government to monitor risks and threats in the innovation sector, as well as to adjust existing regulatory and strategic documents and improve mechanisms to ensure sustainable regional innovative development.
\end{abstract}

Keywords: innovation process; sustainable innovative development; regional innovation policy; indicators for evaluation; resource-type regions.

The presented research was funded by Krasnoyarsk Regional Fund of Science in the framework of the scientific project: «Methodological approaches to the formation of a new system for innovative investment sustainability for resource-type regions based on elaboration of the cluster policy mechanisms (as exemplified by the Krasnoyarsk territory).

Research area: economics.

Citation: Ruiga, I.R. (2021). Empirical assessment of the efficiency of sustainable innovative development of the resource-based regions. J. Sib. Fed. Univ. Humanit. soc. sci., 14(12), 1863-1872. DOI: 10.17516/1997$1370-0865$

(C) Siberian Federal University. All rights reserved

* Corresponding author E-mail address: irina_rouiga@bk.ru 


\title{
Эмпирическая оценка эффективности устойчивого инновационного развития регионов ресурсного типа
}

\author{
И.Р. Руйга \\ Сибирский федеральныий университет \\ Российская Федерачия, Красноярск
}

\begin{abstract}
Аннотация. Статья посвящена формированию методического инструментария оценки для мониторинга рисков и угроз инновационного развития субъектов Российской Федерации с позиции критериев эффективности и устойчивости функционирования. С ориентацией на изученные подходы, с учетом выявленных преимуществ и недостатков автором сформирована методика оценки эффективности инновационного развития регионов с позиции устойчивости. Механизм оценки основан на расчете интегрального индекса по двум проекциям (инновационный потенциал и эффективность инновационного развития). Апробация предложенного методического подхода реализована на примере регионов ресурсного типа Российской Федерации. Результаты исследования могут быть использованы региональными органами государственной власти для мониторинга рисков и угроз в инновационной сфере, а также с целью корректировки действующих нормативно-правовых, стратегических документов и совершенствования механизмов обеспечения устойчивого инновационного развития регионов.
\end{abstract}

Ключевые слова: инновационный процесс, устойчивое инновационное развитие, региональная инновационная политика, оценочные индикаторы, регионы ресурсного типа.

Исследование выполнено при финансовой поддержке краевого государственного автономного учреждения «Красноярский краевой фонд поддержки научной и научно-технической деятельности» в рамках научного проекта № 2019051705126 «Методологические подходы к формированию системы обеспечения инновационноинвестиционной устойчивости для регионов ресурсного типа на основе совершенствования механизмов реализации кластерной политики (на примере Красноярского края)».

Научная специальность: 08.00.00 - экономические науки.

\section{Введение}

В современных условиях приоритетной задачей любого государства выступает обеспечение устойчивого социальноэкономического развития. Масштабные преобразования мировой экономики свидетельствуют о возрастающей роли инновационной деятельности, которая в условиях быстро развивающейся внешней среды, роста конкуренции, а также глобализации становится одним из основных направлений деятельности государства, ориентирующих его на непрерывное и стабильное экономическое развитие, а также на улучшение ключевых аспектов его компетентности.

Новые реалии современной экономики создают необходимость рассмотрения функциональных задач инновационной деятельности в совокупности со стратегическими целями государства, что актуализирует построение эффективно функционирующей национальной инновационной системы государства на двух уровнях: федеральном и региональном. 
На современном этапе формирование региональных инновационных систем остается медленным и противоречивым процессом, что предопределяет необходимость разработки мероприятий и предложений, способствующих повышению устойчивости и эффективности их функционирования, и вызывает теоретико-методический и практический интерес к проведению дальнейшего исследования. Средством повышения устойчивости и эффективности функционирования региональных инновационных систем является совершенствование действий государственных органов власти по формированию институциональных основ, разработке действенного методического инструментария, позволяющего впоследствии корректировать функционирование государственного механизма в области реализации региональной инновационной политики, что обуславливает необходимость методического сопровождения оценки эффективности устойчивого инновационного развития.

\section{Актуальность исследования}

Актуальность исследования вопросов устойчивого инновационного развития повышается в условиях международных экономических санкций, а также высокой дифференциации регионов России по уровню социально-экономического развития. Для неоднородной пространственной системы, которой является Российская Федерация, региональный аспект обеспечения устойчивости развития в инновационной сфере особенно актуален. Особое внимание при этом должно быть уделено регионам ресурсного типа (сырьевым регионам), которые, как правило, являются еще и экспортноориентированными региональными хозяйствами, в связи с чем в первую очередь активно реагируют на внешние глобальные вызовы, при этом осуществляя свою деятельность в соответствии с экспортносырьевой моделью в ущерб повышению эффективности функционирования инновационной сферы.

В связи с этим целью данного исследования выступает формирование методического инструментария оценки для мони- торинга угроз инновационного развития субъектов Российской Федерации с позиции критериев эффективности и устойчивости функционирования.

Для целей данного исследования предлагается использовать понятие «инновационная устойчивость региона» (Ruiga, Stupina, Kovzunova, Chayka, Shkradyuk, 2019). Для определения соответствующей системы показателей и методов количественной оценки эффективности инновационного развития регионов с позиции устойчивости целесообразно взять за основу методологию оценки эффективности инновационной деятельности на федеральном и субфедеральном уровнях.

\section{Методология}

В аспекте международной практики (Bortnik, Senchenya, Mikheeva, Zdunov, Kadochnikov, Sorokina, 2012; Crossing the next regional frontier: Information and Analytics Linking Regional Competitiveness to Investment in a Knowledge Based Economy, 2009; Hollanders, Tarantola, Loschky, 2009; Klowden, Wolfe, 2012; Peran, Sirilli, 2008) система количественной оценки уровня инновационного развития территорий основана на формировании специальных интегральных показателей инновационной сферы. Как правило, поэлементная структура интегрального показателя характеризуется объединением как ресурсных параметров инновационной деятельности, так и результатов ее эффективности. С другой стороны, с точки зрения определения уровня устойчивости инновационного развития наиболее показательным может быть метод сравнения фактических индикаторов инновационного развития с установленными эталонными параметрами. Более того, в аспекте обеспечения состояния устойчивости обязательным условием служит как соответствие пороговому значению, так и улучшение выборки показателей в динамике.

В целом формирование системы пороговых значений широкое распространение получило при определении уровня экономической безопасности (Illarionov, 1998; 
Oleinikov, 2004; Senchagov, 2012), а также уровня устойчивости (Azapagic, 2004; Krajnc, Glavic, 2005; Ruiga, Rogozinskiy, Yamschikov, Kovzunova, Teterin, 2019; Suresh, Erinjery, Jegathambal, 2016; Tan, Lu, 2016; Veisi, Liaghati, Alipour, 2016; Zhao, Chai 2016) социально-экономических систем. Если значение показателя не входит в «зону безопасности», то можно говорить о наличии рисковой ситуации (угрозы), определяемой данным показателем.

Таким образом, опираясь на теорию экономической безопасности и устойчивости развития социально-экономических систем, по мнению автора, целесообразно определить критерии и показатели развития на региональном уровне, а также допустимые (эталонные) их значения, соответствие которым дает основание утверждать о наличии устойчивого развития (Vissarionov, Gumerov, 2017).

Обобщая преимущества и недостатки научных подходов к оценке инновационного развития и идентификации угроз в иннова- ционной сфере (следуя теории экономической безопасности), наиболее обоснованной с точки зрения практической реализации, по мнению автора, представляется методика на основе формирования интегрального индекса в рамках двух проекций (Ruiga, Stupina, Kovzunova, Chayka, Shkradyuk, 2019): 1) инновационный потенциал региона; 2) результаты инновационной деятельности региона (эффективность инновационного развития). Интегральные индексы (или показатели) формируются с учетом частных показателей, которые предварительно подвергаются простому нормированию на величину расхождения между их фактическими и пороговыми значениями. Это позволяет обеспечить оценку инновационного развития с позиции устойчивости. Пороговые значения сформированы с учетом текущих международных тенденций.

Последовательность этапов методического инструментария оценки эффективности инновационного развития регионов с позиции устойчивости представлена на рис. 1 .

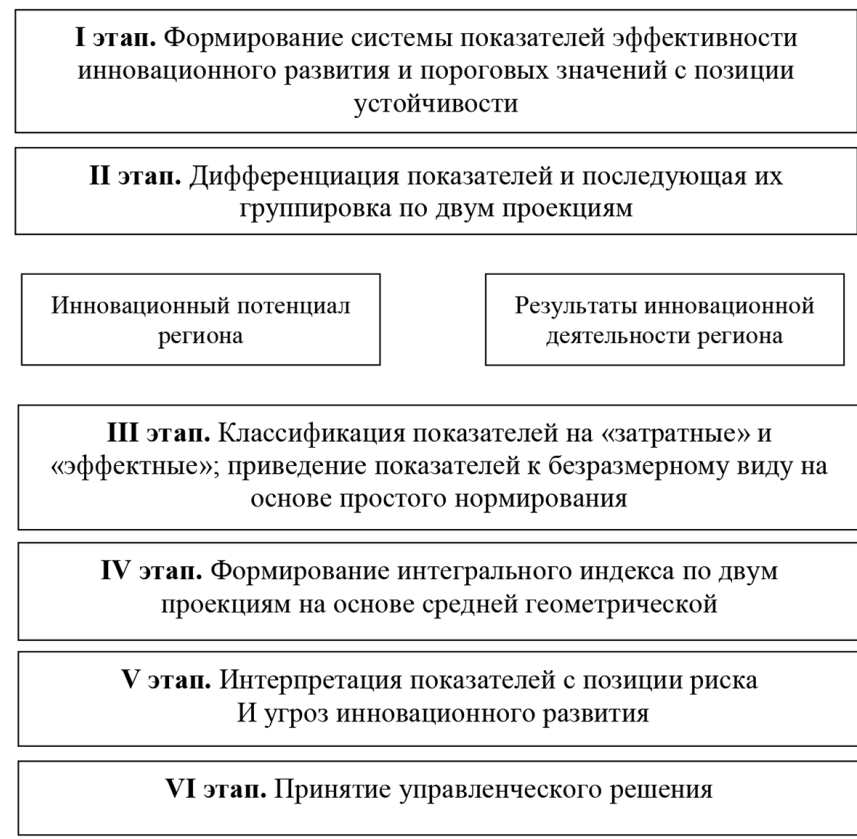

Рис. 1. Алгоритм оценки эффективности инновационного развития регионов с позиции устойчивости (составлено автором)

Fig. 1. Algorithm for assessing the effectiveness of innovative development of regions from the standpoint of sustainability (compiled by the author) 
В рамках первого и второго этапов формируется система показателей для проведения процедуры мониторинга исходя из стратегических целей регионального инновационного развития. Система оценочных индикаторов и пороговых значений была скорректирована с учетом наличия соответствующих статистических данных (табл. 1).

В рамках третьего этапа в соответствии с предложенным алгоритмом оценки все показатели подразделяются на две группы (Mitiakov, Kornilov, 2015): группа показателей, пороговое значение для которых является минимально допустимым уровнем для обеспечения устойчивого инновационного развития региона (минимальное пороговое значение); группа показателей, пороговое значение для которых является максимально допустимым уровнем для сохранения устойчивого инновационного развития региона (максимальное пороговое значение). Показатели первой группы условимся называть «эффектными» показателями. Рост данных показателей будет приводить к повышению уровня инновационного развития региона. Показатели второй группы, т. е. те, чей рост будет приводить к ухудшению уровня инновационного развития, будем считать «затратными» показателями.

Далее осуществляется приведение показателей к безразмерному виду методом простого нормирования. Это обусловлено тем, что показатели, входящие в систему, измеряются в разных единицах (процентах, разах, рублях, тоннах и пр.), т. е. имеют разную размерность, которая затрудняет совместное отображение и анализ выбранных показателей. Нормировка приводит к безразмерности показателей при сохранении структуры изменения исходных значений. После приведения показателей к безразмерному виду возможно представить и анализировать их в единой системе координат.

Таким образом, для «эффектных» показателей следует использовать вид функции соотношения фактического значения показателя и порогового, представленный

Таблица 1. Скорректированная система показателей и пороговых значений для оценки эффективности инновационного развития региона с позиции устойчивости (составлено автором по данным (Ruiga, Stupina, Kovzunova, Chayka, Shkradyuk, 2019)

Table 1. Adjusted system of indicators and threshold values for assessing the effectiveness of innovative development of the region from the standpoint of sustainability (compiled by the author based on data from (Ruiga, Stupina, Kovzunova, Chayka, Shkradyuk, 2019)

\begin{tabular}{|l|c|}
\hline \multicolumn{1}{|c|}{ Показатель } & Пороговое значение \\
\hline \multicolumn{1}{|c|}{ Инновационный потенциал (Ресурсы инновационного развития) } \\
\hline $\begin{array}{l}\text { Численность персонала, занятого научными исследованиями и разработками } \\
\text { в общей численности занятых в регионе, } \%\end{array}$ & не менее 5 \\
\hline Доля внутренних затрат на исследования и разработки в объеме ВРП, \% & не менее 2 \\
\hline Доля инвестиций в основной капитал в объеме ВРП, \% & не менее 25 \\
\hline $\begin{array}{l}\text { Доля организаций, осущестлявших технологические инновации, в общем числе } \\
\text { обследованных региональных организаций, \% } 30\end{array}$ & не более 40 \\
\hline Износ основных производственных фондов, \% & не менее 15 \\
\hline $\begin{array}{l}\text { Объем инновационных товаров, работ, услуг в общей доле отгруженных товаров } \\
\text { региона, \% }\end{array}$ & не менее 6 \\
\hline Число выданных патентов на 10000 чел. населения региона, ед. & не менее 5 \\
\hline $\begin{array}{l}\text { Число отечественных патентных заявок на изобретения, поданных в регионе, } \\
\text { в расчете на 10000 чел. населения, ед. }\end{array}$ & не менее 5 \\
\hline $\begin{array}{l}\text { Соотношение объема отгруженной инновационной продукции и затрат } \\
\text { на технологические инновации, коэффициент }\end{array}$ & ненойделельности \\
\hline
\end{tabular}


в формуле (1), а для «затратных» показателей - представленный в формуле (2):

$$
y=x / x_{0}, y>1
$$

где $y$ - значение «эффектного» показателя; $x$ - фактическое значение показателя; $x_{0}-$ пороговое значение показателя;

$$
y=x_{0} / x, y>1
$$

где $y$ - значение «затратного» показателя; $x$ - фактическое значение показателя; $x_{0}-$ пороговое значение показателя.

Из представленных формул $(1,2)$ следует, что значение «эффектного» или «затратного» показателя ниже единицы свидетельствует о необходимости идентификации угрозы в развитии инновационной сферы региона.

В рамках четвертого этапа осуществляется расчет интегрального индекса (показателя), характеризующего уровень инновационного развития региона, вычисление которого производится по каждой группе показателей в случае их дифференциации по определенным проекциям по формуле (3),

$$
I=\sqrt[n]{\sum_{i=1}^{n} f_{i}}, i=\overline{1, n},
$$

где $I$ - интегральный индекс (показатель), характеризующий состояние инноваци- онного развития в конкретной проекции; $f_{i}$ - нормированное значение і-го показателя в системе; $n$ - количество показателей в системе.

В рамках заключительного этапа по результатам оценки в зависимости от целевых ориентиров принимается управленческое решение по нивелированию и противодействию выявленных рисков и угроз инновационного развития, а также корректировке инструментов и методов реализуемой инновационной политики региона.

\section{Результаты исследования}

Апробация предложенного методического подхода произведена на совокупности регионов ресурсного типа Российской Федерации. Выборка регионов осуществлялась на основе показателей «Инвестиции в основной капитал в отрасль «Добыча полезных ископаемых» и «Коэффициент локализации» добывающих отраслей в регионе». По указанным направлениям сформированы соответствующие рейтинги регионов, на основании которых произведен расчет средней геометрической для формирования итогового значения, который впоследствии заложен в разработку рейтинга регионов «ресурсного типа» (табл. 2).

\begin{tabular}{|c|c|c|c|c|c|}
\hline $\begin{array}{c}\text { Субъект } \\
\text { Российской Федерации } \\
\end{array}$ & $\begin{array}{c}\text { Сводный } \\
\text { индекс }\end{array}$ & Ранг & $\begin{array}{c}\text { Субъект } \\
\text { Российской Федерации } \\
\end{array}$ & \begin{tabular}{c|} 
Сводный \\
индекс \\
\end{tabular} & Ранг \\
\hline $\begin{array}{l}\text { Ханты-Мансийский автономный } \\
\text { округ - Югра }\end{array}$ & 1,41 & 1 & Иркутская область & 12,25 & 11 \\
\hline Ненецкий автономный округ & 2,45 & 2 & Республика Татарстан & 12,49 & 12 \\
\hline $\begin{array}{l}\text { Ямало-Ненецкий } \\
\text { округ }\end{array}$ & 2,83 & 3 & Чукотский автономный округ & 12,73 & 13 \\
\hline Сахалинская область & 3,00 & 4 & Пермский край & 13,67 & 14 \\
\hline Республика Саха (Якутия) & 6,32 & 5 & Астраханская область & 14,00 & 15 \\
\hline Кемеровская область & 7,07 & 6 & Магаданская область & 14,70 & 16 \\
\hline Республика Коми & 7,48 & 7 & Удмуртская Республика & 15,91 & 17 \\
\hline Оренбургская область & 7,94 & 8 & Самарская область & 17,32 & 18 \\
\hline Красноярский край & 8,00 & 9 & Мурманская область & 17,49 & 19 \\
\hline Томская область & 10,82 & 10 & Белгородская область & 19,97 & 20 \\
\hline
\end{tabular}

Таблица 2. Рейтинг регионов «ресурсного типа» (Топ-20) *

Table 2. Rating of regions of the «resource type» (Top-20)

* Составлено автором на основании расчетов по данным Росстата. 
Автором сознательно из данного перечня исключены Ненецкий и Чукотский автономные округа ввиду отсутствия большого объема официальной статистики в разрезе инновационной составляющей. Таким образом, выборка «ресурсных регионов» будет представлена 18-ю субъектами Российской Федерации. Временной период для оценки: 2007-2016 гг. (с учетом доступности статистических данных). Результаты апробации предложенной методики представлены в соответствии с табл. 3,4 .

В целом, важно отметить, что уровень инновационного развития с позиции устойчивости регионов России ресурсного типа остается на достаточно низком уровне в течение всего рассматриваемого периода, что обусловлено небезопасным состоянием факторов, влияющих на устойчивость региональной инновационной системы. Оценка частных показателей, входящих в состав интегрального индекса региона, выявила, что практически по всем показателям наблюдается существенное отклонение фактических значений от установленных пороговых. С другой стороны, нужно понимать, что формирование пороговых значений в данном случае осуществлялось исходя из мировых тенденций инновационного развития.

По результатам оценки по двум проекциям наиболее устойчивыми в аспекте инновационного развития являются Томская и Самарская области, Республика Татарстан, Пермский край. Во многом на значение интегральной оценки повлияли

Таблица 3. Рейтинг регионов ресурсного типа по интегральному показателю «Инновационный потенциал» *

Table 3. Rating of resource-type regions by the integral indicator «Innovation potential»

\begin{tabular}{|c|c|c|c|c|c|}
\hline \multirow{2}{*}{$\begin{array}{c}\text { Регионы } \\
\text { ресурсного типа }\end{array}$} & \multicolumn{3}{|c|}{ Рейтинг за 2016 г. } & \multicolumn{2}{|c|}{$\begin{array}{l}\text { Рейтинг по среднему } \\
\text { значению Іп за } 10 \text { лет }\end{array}$} \\
\hline & Іп & $\begin{array}{c}\text { Ранг } \\
\text { среди регионов } \\
\text { ресурсного типа }\end{array}$ & $\begin{array}{c}\text { Ранг } \\
\text { среди всех } \\
\text { регионов России }\end{array}$ & Іп & Ранг \\
\hline Томская область & 0,650 & 1 & 7 & 0,71 & 1 \\
\hline Республика Татарстан & 0,508 & 2 & 10 & 0,50 & 4 \\
\hline Пермский край & 0,436 & 3 & 22 & 0,49 & 5 \\
\hline Магаданская область & 0,423 & 4 & 25 & 0,57 & 2 \\
\hline Красноярский край & 0,408 & 5 & 27 & 0,43 & 6 \\
\hline Мурманская область & 0,372 & 6 & 30 & 0,40 & 7 \\
\hline Республика Саха & 0,338 & 7 & 35 & 0,37 & 8 \\
\hline Самарская область & 0,331 & 8 & 36 & 0,50 & 3 \\
\hline Республика Коми & 0,329 & 9 & 37 & 0,35 & 10 \\
\hline Иркутская область & 0,287 & 10 & 50 & 0,37 & 9 \\
\hline Белгородская область & 0,279 & 11 & 51 & 0,28 & 12 \\
\hline Астраханская область & 0,230 & 12 & 61 & 0,29 & 11 \\
\hline Сахалинская область & 0,224 & 13 & 66 & 0,25 & 13 \\
\hline Удмуртская Республика & 0,219 & 14 & 69 & 0,25 & 14 \\
\hline Ханты-Мансийский АО & 0,184 & 15 & 76 & 0,20 & 15 \\
\hline Оренбургская область & 0,178 & 16 & 77 & 0,19 & 16 \\
\hline Кемеровская область & 0,171 & 17 & 79 & 0,19 & 17 \\
\hline Ямало-Ненецкий АО & 0,097 & 18 & 85 & 0,08 & 18 \\
\hline
\end{tabular}

* Рассчитано автором по данным Росстата. 
Irina R. Ruiga. Empirical Assessment of the Efficiency of Sustainable Innovative Development...

Таблица 4. Рейтинг регионов ресурсного типа по интегральному показателю «Результаты инновационной деятельности» *

Table 4. Rating of resource-type regions according to the integral indicator «Results of innovation activity»

\begin{tabular}{|c|c|c|c|c|c|}
\hline \multirow[b]{2}{*}{ Регионы ресурсного типа } & \multicolumn{3}{|c|}{ Рейтинг за 2016 год } & \multicolumn{2}{|c|}{$\begin{array}{l}\text { Рейтинг по среднему } \\
\text { значению Іп за } 10 \text { лет }\end{array}$} \\
\hline & Іп & \begin{tabular}{|c|} 
Ранг \\
среди регионов \\
ресурсного типа
\end{tabular} & $\begin{array}{c}\text { Ранг } \\
\text { среди всех } \\
\text { регионов России }\end{array}$ & Іп & Ранг \\
\hline Республика Татарстан & 0,651 & 1 & 4 & 0,66 & 1 \\
\hline Самарская область & 0,517 & 2 & 10 & 0,63 & 2 \\
\hline Удмуртская Республика & 0,515 & 3 & 11 & 0,27 & 6 \\
\hline Пермский край & 0,483 & 4 & 13 & 0,45 & 3 \\
\hline Томская область & 0,373 & 5 & 25 & 0,41 & 4 \\
\hline Белгородская область & 0,285 & 6 & 31 & 0,35 & 5 \\
\hline Республика Саха & 0,264 & 7 & 34 & 0,15 & 12 \\
\hline Астраханская область & 0,256 & 8 & 35 & 0,18 & 9 \\
\hline Кемеровская область & 0,249 & 9 & 37 & 0,18 & 10 \\
\hline Красноярский край & 0,233 & 10 & 40 & 0,20 & 8 \\
\hline Республика Коми & 0,201 & 11 & 46 & 0,22 & 7 \\
\hline Оренбургская область & 0,175 & 12 & 56 & 0,15 & 13 \\
\hline Иркутская область & 0,135 & 13 & 64 & 0,13 & 15 \\
\hline Мурманская область & 0,128 & 14 & 65 & 0,08 & 16 \\
\hline Магаданская область & 0,062 & 15 & 75 & 0,14 & 14 \\
\hline Ямало-Ненецкий АО & 0,056 & 16 & 79 & 0,07 & 17 \\
\hline Ханты-Мансийский АО & 0,032 & 17 & 83 & 0,05 & 18 \\
\hline Сахалинская область & $0,011 * *$ & 18 & 85 & 0,15 & 11 \\
\hline
\end{tabular}

* Рассчитано автором по данным Росстата.

** В 2016 г. для расчета интегрального показателя «Результаты инновационной деятельности» в Сахалинской области не был взят показатель «Объем инновационных товаров, работ, услуг в общей доле отгруженных товаров региона», так как его значение равно 0.

показатели инновационной деятельности: внутренние расходы на НИОКР, затраты на технологические инновации.

Наиболее уязвимыми к рискам и угрозам инновационного развития к концу 2016 г. являлись Ханты-Мансийский и Ямало-Ненецкий автономные округа. В числе основных причин, обеспечивающих низкие позиции в рейтинге, присутствуют высокая изношенность основных фондов и слабая инновационная активность, проявляющаяся в очень низком объеме произведенной продукции в сфере инноваций.

\section{Заключение}

По мнению автора, методика оценки эффективности инновационного развития регионов ресурсного типа с позиции устойчивости направлена на совершенствование инструментов исследования тенденций и проблем развития в инновационной сфере и может формировать основу для корректировки действующих и создания новых механизмов управления сбалансированным инновационным развитием региона и обеспечения необходимых условий реализации приоритетных направлений пространственного социально-экономического развития. 
Методика апробирована на примере регионов ресурсного типа и позволяет:

- проводить процедуру регулярного мониторинга инновационного развития субъектов ресурсного типа с учетом целевых показателей и международных тенденций;

- определять соответствие региональных ресурсов инновационного развития результатам инновационной деятельности в субъектах Российской Федерации;

- идентифицировать риски и угрозы инновационного развития регионов;

- формировать практические рекомендации по нивелированию и противодействию выявленных рисков и угроз инновационного развития, а также корректировки инструментов и методов реализуемой инновационной политики региона.

Таким образом, выявленные угрозы формируют основу для принятия управленческих решений со стороны государственных органов власти в части изучения положительных практик в аспекте устойчивого инновационного развития регионов ресурсного типа (Республика Татарстан, Пермский, край, Самарская и Томская области) с целью совершенствования существующих инструментов и методов и формирования нового эффективного инструментария в аспекте регулирования и стимулирования инновационной деятельности.

\section{Список литературы / References}

Azapagic, A. (2004). Developing a framework for sustainable development indicators for the mining and minerals industry, In Journal of Cleaner Production, 12, 639-62. DOI: 10.1016 /S0959-6526(03)00075-1.

Bortnik, I. M., Senchenya, G.I., Mikheeva, N.N., Zdunov, A.A., Kadochnikov, P.A., Sorokina, A.V. (2012). Sistema otsenki I monitoringa innovatsionnogo razvitiia regionov Rossii [System of assessment and monitoring of innovative development of Russian regions], In Innovatsii [Innovations] 9(176), 48-61.

Crossing the next regional frontier: Information and Analytics Linking Regional Competitiveness to Investment in a Knowledge Based Economy, U. S. Economic Development Administration (2009). Available at: http://www.statsamerica.org/innovation.

Hollanders, H., Tarantola, S., Loschky, A. (2009). Regional Innovation Scoreboard (RIS), In Pro Inno Europe, available at: http://www. proinno-europe.eu/page/regional-innovation-scoreboard.

Illarionov, A. (1998). Kriterii ekonomicheskoi bezopasnosti [Criteria of economic security], In Voprosy ekonomiki [Issues of Economics] 10, 35-58.

Klowden, K., Wolfe, M. (2012). State Technology and Science Index. Enduring Lessons for the Intangible Economy (Milken Institute). Available at: http:// www.milkeninstitute.org/pdf/STSI2013.pdf.

Krajnc, D., Glavic, P. (2005). A model for integrated assessment of sustainable development, In Resources, Conservation and Recycling, 43, 189-208. DOI: 10.1016/j.resconrec.2004.06.002.

Mitiakov, E.S., Kornilov, D.A. (2015). K voprosu o vybore vesov pri nahozhdenii integral'nyh pokazatilei economicheskoi dinamiki [Regarding the issue of proper weighting coefficients in determination of integral indicators of economic dynamics], In Ekonomika, innovatsii I menedzhment [Economics, innovations and management] 2, 112.

Oleinikov, E.A. (2004). Economicheskaia i natsionalnaia bezopasnost' [Economic and National Security]. Moscow, Exam.

Peran, L., Sirilli, P. (2008). Benchmarking of innovation activity of Europian countries, In Foresight, $1(5), 4-15$.

Ruiga, I.R., Rogozinskiy, E.V., Yamschikov, A.S., Kovzunova, E.S., Teterin, Yu.A. (2019). Metodological aspects of machine-buildig complex economic security from the position of innovative and investment sustainability: macroeconomic cross-section, In Journal of Physics: Conference Series, 1399, 033088. DOI: 10.1088/1742-6596/1399/3/033088.

Ruiga, I.R., Stupina, A.A., Kovzunova, E.S., Chayka, A.A., Shkradyuk, I.A. (2019). Practical implementation of data Envelopment Analysis technology to asses the innovative sustainability of resource-type regions, In In Journal of Physics: Conference Series, 1399, 033118. DOI: 10.1088/1742-6596/1399/3/033118. 
Senchagov, V.K. (2012). Economicheskaia bezopasnost' regionov Rossii [Economic Security of Russian Regions]. Nizhny Novgorod.

Suresh, B., Erinjery, J.J., Jegathambal, P. (2016). Indicators and Influence Factors for Sustainability Assessment of Inclusive Smart Innovation Clusters, In Journal of Geological Resource and Engineering, 7, 305-27. DOI: $10.17265 / 2328-2193 / 2016.07 .001$.

Tan, F. Lu, Z. (2016). Assessing regional sustainable development through an integration of nonlinear principal component analysis and Gram Schmidt orthogonalization, In Ecological Indicators, 63, 71-81.

Veisi, H., Liaghati, H., Alipour, A. (2016). Developing an ethics-based approach to indicators of sustainable agriculture using analytic hierarchy process (AHP), In Ecological Indicators, 60, 644-54.

Vissarionov, A.B., Gumerov, R.R. (2017). Ob ispol'zovanii predel'nykh (porogovykh) znachenii indikatorov ekonomicheskoi bezopasnosti Rossiiskoi Federatsii [On the use of limit (threshold) values of indicators of economic security of the Russian Federation], In Sthategicheskii menedzhment [Management Sciences] 3, 12-20.

Zhao, J., Chai, L. (2016). A Novel Approach for Assessing the Performance of Sustaiable Urbanization Based on Structural Equation Modeling: A China Case Study, In Sustainability, 8(9), 910. DOI: 10.3390/ su8090910. 